\title{
ArTSchives: un tremplin vers divers types de musées et vers la création artistique
}

\author{
Lise Robichaud, Moncton University \\ lise.r.robichaud@umoncton.ca
}

Résumé L'article présente un modèle d'enseignement des arts visuels intitulé arTSchives qui intègre les notions de musée, d'archives et d'apprentissage expérientiel. Le but est de faciliter la compréhension des œuvres d'art contemporain de genre archivistique tout en proposant des pistes de création artistique intégrant le concept d'archives. L'auteure a procédé par une analyse de contenu d'installations en arts visuels ce qui a mené à l'énumération d'une liste d'exemples de sources d'archives possibles. Ce type particulier d'enseignement encourage la fréquentation des musées d'art contemporain ainsi que des musées de sciences naturelles, sciences humaines et autres musées spécialisés.

Mots clés: Contemporain; musées; modèle d'enseignement; contexte culturel acadien; appréciation artistique; art archivistique; création artistique; galerie d'art.

\section{Introduction}

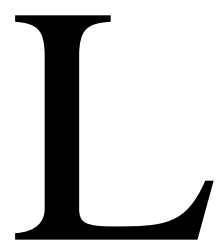

e domaine des arts visuels, par la variété de ses productions artistiques, contribue à la découverte de notre propre culture et de celles des autres. Cela se concrétise par la fréquentation des œuvres d'art exposées dans les Musées, et autres lieux de diffusion de l'art, ainsi que par l'éducation artistique et esthétique. Or, l'art offre certains défis de lecture. C'est le cas, par exemple, pour le public canadien lorsqu'il va à la rencontre de l'art contemporain (Lachapelle 2013). Nous avons observé ce même phénomène dans les cours de Didactique des arts visuels que nous enseignons au niveau de la formation des maitres ainsi que dans notre pratique d'artiste professionnelle en arts visuels.

Dans le cadre de divers symposiums en arts visuels dans l'est du Canada, alors que nous faisions de l'art en direct, on a pu constater la volonté du public de se rapprocher du sens de l'œuvre d'art contemporain. À titre d'exemple, nous avons observé qu'il y avait une curiosité pour les œuvres d'art contemporain incorporant différentes sources d'archives et faisant appel à la participation du public. Ce même désir d'apprendre, en particulier à propos de l'art 
archivistique, nous l'avons observé chez nos étudiantes et étudiants. C'est ce concept d'archives que nous avons retenu pour développer le modèle d'enseignement des arts visuels résumé dans cet article.

Pour ce faire, nous approchons le sujet à titre d'artiste-enseignante-acadienne, c'est pourquoi il sera question du contexte francophone néo-brunswickois avant d'aborder le sujet de l'activité d'approche de l'œuvre d'art. Puis, afin de cerner le concept de l'archivistique, nous présenterons quelques exemples de caractéristiques d'art archivistiques qui furent dégagés à partir de l'analyse de nos propres productions en art contemporain. Une vision du musée sera présentée, suivie du modèle d'enseignement ancré dans la perspective archivistique. Pour terminer, quelques exemples de résultats d'essais exploratoires de ce modèle d'enseignement seront cités.

\section{Contexte culturel acadien}

Artiste et professeure de didactique des arts visuels à l'Université de Moncton, nous préparons les futurs enseignants et enseignants du primaire à enseigner les arts visuels de la maternelle à la huitième année en milieu francophone minoritaire de l'est du Canada. De plus, nous donnons le cours de didactique des arts visuels au secondaire pour les personnes ayant un baccalauréat en arts visuels qui viennent se spécialiser en éducation dans le cadre d'un baccalauréat spécial en éducation d'une durée de deux ans. La formation en enseignement des arts visuels étant ainsi structurée, nous devons développer du matériel pédagogique propre aux besoins de ce contexte à la fois pour le volet création et appréciation artistique.

L'idée d'un modèle d'enseignement des arts visuels dans une perspective archivistique nous est venue dans le cadre d'expériences pratiques sur le terrain que nous menons chaque année dans les cours de Didactique des arts visuels. Depuis 1988, ces expériences sont menées 
sur le terrain du Musée acadien (http://www.umoncton.ca/umcm-maum/) et de la Galerie d'art Louise-et-Reuben-Cohen de l'Université de Moncton (http://www.umoncton.ca/umcm-ga/) afin de faire des liens entre la théorie et la pratique. Situés dans l'Édifice Clément-Cormier, le Musée présente des objets témoins de l'histoire du peuple acadien alors que la Galerie se spécialise dans la diffusion de l'art contemporain de l'Acadie et d'ailleurs. Ce sont deux lieux de diffusion de la culture acadienne et francophone.

Cette identité est d'ailleurs incorporée au secteur de l'éducation. Au Nouveau-Brunswick le Ministère de l'Éducation et de la petite Enfance a une section anglophone et une section francophone. Ainsi, les acadiens et autres francophones de la province ont donc leurs propres programmes d'études en arts visuels et les deux résultats d'apprentissages généraux sont l'éducation artistique et l'éducation esthétique. Ces programmes furent analysés sous différents angles qu'il s'agisse de l'angle historique (Robichaud 1990), arts médiatiques (Robichaud 2004) ou mathématiques (Robichaud 2011a). Cependant, les documents de ces programmes étant toujours ancrés dans le paradigme moderne (ex. contenu axé sur les notions du langage plastique), une mise à jour s'impose afin d'en arrimer le contenu avec l'art du 21e siècle et ses caractéristiques. Une telle mise à jour permettrait de faciliter la lecture d'œuvres d'art contemporain archivistique d'artistes acadiens du Nouveau-Brunswick comme Marjolaine Bourgeois, Mario Doucette Mathieu Léger et Paul Édouard Bourque, pour ne nommer que ceuxlà, et de tous les autres artistes professionnels et professionnelles qui pratiquent en art contemporain.

Ainsi, aux notions d'outils et matériaux, de procédés techniques, de langage plastique et d'histoire qu'on propose dans les programmes d'études en arts visuels certains concepts s'imposent afin de se mettre à jour avec l'art de notre époque. Il s'agit, par exemple, des 
concepts de métaphore, de contexte, d'éphémère, du ludique, du médiatique et surtout de l'archivistique. Ainsi, le matériel pédagogique en enseignement des arts visuels faciliterait la préparation des apprenantes et des apprenants au décodage des œuvres d'art contemporain qui sont diffusées dans les Musées, des lieux qui regorgent d'archives.

\section{Musée et culture visuelle.}

Médiateur culturel de l'héritage matériel, le musée est à la fois un lieu éducatif et un lieu de rêverie avec ses objets visuels qu'ils soient réels ou virtuels. Quelque soit la catégorie de musée, la plupart des musées du monde sont aujourd'hui branchés avec les nouvelles technologies (Lopez, Margapoti, Maragliano et Bove 2010), et cela, qu'il s'agisse de musées d'art contemporain, de musées d'art décoratif, de musées de sciences naturelles (incluant les jardins botaniques et planétariums), de musées de sciences humaines (sciences sociales, historiques et technologiques) ou de musées spécialisés (ex. souliers, chocolat, équipe de soccer, cadenas, voitures, etc.). En fait, on parle ici de culture visuelle, pour reprendre un terme de Duncum (2001). Pour les artistes ces lieux de diffusion de la culture forment l'une des maintes sources d'inspirations possibles.

Dans l'Acadie du Nouveau-Brunswick (Canada), la culture visuelle des Musées comprend entre autres les artéfacts en lien avec la culture acadienne. On en retrouve au Musée acadien de l'Université de Moncton ainsi qu'au village historique acadien de Caraquet, un endroit qui fait revivre les costumes, l'architecture et les coutumes du peuple acadien au $18^{\mathrm{e}}$ siècle jusqu'au début du $20^{\mathrm{e}}$ siècle. Vers la fin des années 1970 , nous y avons travaillé en tant que guide interprète de la Maison Martin $\left(18^{\mathrm{e}} \mathrm{s}\right)$.

Cette expérience sur le terrain d'un musée « vivant» a contribué à forger notre identité culturelle et a influencé notre art, car les archives identitaires et historiques sont souvent 
présentes dans nos œuvres. Celles-ci explorent parallèlement, et parfois conjointement, l'art pictural et la sculpture éphémère d'une manière poétique et autobiographique. Les problématiques sont de nature intime et sociale comme l'identité et la culture.

\section{De l'écriture à la lecture.}

Il existe dans la littérature spécialisée différents modèles de lecture de l'art contemporain. Celle de Feinstein (1989) avec le concept de métaphore nous interpelle. C'est peut-être parce qu'en Acadie plusieurs artistes en arts visuels sont aussi reconnus en tant que poètes (ex. Daniel Dugas, Roméo Savoie, Herménégilde Chiasson). Mais c'est surtout parce que dans nos cours de didactique des arts visuels au primaire et au secondaire on fait appel à la création littéraire (ex. poésie) dans le processus de lecture de l'œuvre. Nous avons constaté par la pratique que la poésie est un moyen à notre portée et efficace pour amener les apprenantes et les apprenants à se rapprocher du sens de l'œuvre d'art d'une manière personnelle et à vivre une expérience esthétique unique. Il en ressort que l'œuvre prend un nouveau sens pour le regardeur.

Cette approche intégrant l'usage de la métaphore est valorisée par des chercheurs spécialisés dans l'approche critique de l'œuvre d'art en éducation artistique. Il en est ainsi pour White (2014) dont les publications récentes mettent l'attention sur la nature de l'expérience du regardeur et sur le processus de découverte dans l'approche critique de l'œuvre d'art. L'approche pédagogique qu'il propose intègre le concept du devenir. Ainsi, on tient compte des apprenantes et des apprenants dans le processus de l'activité d'appréciation des oeuvres d'art afin de favoriser l'expérience esthétique chez les jeunes.

White (2014) souligne le fait que certaines approches de l'oeuvre d'art peuvent convenir à telle ou telle personne. Il serait donc préférable de prévoir plusieurs alternatives en ce qui 
concerne l'approche de l'oeuvre d'art. Bien qu'il ne s'agit pas de l'unique moyen à proposer, la métaphore (ex. poésie) est l'un des moyens retenus.

Suite à une revue de la littérature, nous avons constaté qu'il ne semble pas exister de stratégie d'enseignement des arts visuels intégrant la notion d'archives et de musée tel que nous l'envisageons. Or, actuellement, en enseignement des arts visuels, il existe un besoin en matière de développement de modèles d'enseignement des arts visuels intégrant le concept de l'archivistique. C'est d'ailleurs ce qui a motivé cet article. Développer un tel modèle nécessite toutefois de présenter certains concepts, dont celui de l'art archivistique.

\section{Quelques caractéristiques de l'art archivistique.}

Nous devons à Foster (2004), l'identification des caractéristiques de l'art contemporain à tendance archivistique. Son analyse révèle que ce sont surtout des installations mettant en scène divers objets provenant de la culture populaire et l'usage des nouvelles technologies. De plus, l'information historique ainsi que l'idée de «collection » y sont souvent présentes physiquement. Cette tendance d'archivistique en arts visuels est actuellement très présente en art contemporain.

Les domaines de la création et l'archivage se côtoient de plus en plus, surtout depuis l'avènement du numérique. Un colloque, sous la direction de Klein, Lacombe et Lemay (2014), nous a amené à contribuer en tant qu'artiste en analysant notre propre production artistique en arts visuels sur une période de dix ans.

Il en est ressorti l'identification de caractéristiques d'archives dont une présence d'archives de type généalogique (ex. origines culturelles), folklorique (ex. la légende du bateau fantôme), littéraire (ex. poésie), mathématique (ex. calcul de l'extension), écologique (ex. déversement de pétrole par l'Exon Valdès), intime (ex. souvenirs de famille) et social (ex. l'actualité). L'analyse de contenu a aussi révélé que le public d'art est parfois une source 
d'archives (ex. lorsque l'artiste recueille des informations auprès du public pour incorporer à son œuvre dans le cadre d'évènement d'art en direct) et que si le matériel est archivé, l'immatériel (ex. échanges verbaux avec l'artiste) ne l'est pas. Une deuxième vérification à l'aide de textes de critiques d'art a donné des résultats similaires. Par exemple, Grande (2000) relève, à propos de l'une de nos installations (Robichaud 2000), des caractéristiques de l'archivistique lorsqu'il écrit "populaire", "historique", "ethnologie" et "généalogie" (p.55). Les résultats de cet exercice d'analyse forment une liste d'exemples d'archives à intégrer au développement d'un modèle d'enseignement des arts visuels dans une perspective archivistique.

\section{Une vision intégrant l'expérimentation et la découverte.}

Par le passé, on a réfléchi sur les liens entre les arts visuels et le musée dans la formation des maitres (Robichaud 2011b; Robichaud 2013). C'est toutefois la première fois qu'on développe un modèle d'enseignement ancré dans la perspective archivistique. Un modèle préconisant l'action puisque par les arts visuels nous communiquons nos idées et nous inventons des solutions esthétiques fabriquées avec de la matière. Et cela, dans un espace soit naturel, artificiel ou virtuel.

Voir des œuvres d'art archivistique, en identifier les caractéristiques et chercher des liens avec des objets qui se trouvent dans d'autres types de musées pour ensuite retourner en atelier et créer sa propre réalisation artistique de genre archivistique, voilà la stratégie proposée au cœur du modèle d'enseignement de l'art dans une perspective archivistique. Les étapes réfèrent à la notion d'apprentissage expérientiel de Kolb (1984) car il y a une expérience pratique, un retour critique pour clarifier ce qui a été appris, une formulation de questions et application à d'autres contextes de création ou d'appréciation. 
Toujours dans cette perspective, nous cherchons à rendre le cours d'arts visuels ludique pour les élèves en proposant un ensemble d'activités pédagogiques interreliées en intégrant des notions de jeu (ex. jeu de la découverte), de réussite (ex. il n'y a pas de mauvaise réponse), de culture vivante (ex. rencontre avec l'artiste), d'expérimentation du regard (ex. voir les œuvres d'art archivistiques en exposition au Musée d'art contemporain) et des étapes du processus de création (ex. vivre une expérience d'atelier en 4 temps : explorer, trouver, créer, regarder). Dans un tel contexte, les élèves participeraient dans un processus tenant compte de leurs idées et suggestions. Il s'agit d'une approche pédagogique qui fonctionne comme un tremplin vers l'œuvre d'art archivistique et vers la création artistique intégrant le concept d'archive. C'est un départ du lieu de diffusion de l'art archivistique vers d'autres types de musées et vice versa. C'est aussi une approche qui fera vivre des expériences esthétiques et artistiques aux jeunes et qui leur permettra de développer le gout de la fréquentation d'œuvres d'art contemporain. Pour ce faire, voici le processus méthodologique suivi.

\section{Processus méthodologique}

Méthodologiquement, notre réflexion repose sur la logique des étapes de développement de devis pédagogique. On a opté pour le modèle d'approche réfléchie de Walker (1990) qui propose de débuter par la présentation critique des programmes existants afin d'en déceler les composantes et les carences. Ensuite, il s'agissait de présenter une autre vision d'un devis pédagogique, ce qui fut présenté dans la première partie de l'article. Puis il y eut l'étape d'analyse des concepts qui nous a menés à penser qu'un tel devis est souhaitable. Ces concepts furent ceux de l'archivistique, du musée et de l'éducation. La dernière étape est l'étape réflexive qui aboutit au plan pour transformer ces idées en stratégie d'enseignement utilisable par la personne enseignante et ses élèves. Voici le nom et la forme que nous lui donnons. 


\section{ArTSchives}

Le modèle d'enseignement arTSchives, a pour titre un néologisme issu de la fusion des mots «art » et «archives ». Inspiré de l'apprentissage expérientiel, arTSchives se veut inductif, axé sur l'apprenante ou l'apprenant et au service des activités d'appréciation artistique (au Musée d'art contemporain ou autre lieu de diffusion d'art archivistique) et de création artistique (en atelier ou à l'extérieur sur un terrain vague). L'accent demeure placé sur la démarche plutôt que sur le produit.

L'expérience visée est d'amener l'apprenante ou l'apprenant à découvrir l'art archivistique pour ensuite faire part de ses réactions et observations. On espère que de leur propre analyse sortiront d'autres exemples de caractéristiques d'art archivistiques. À titre d'exemple, des élèves partent à la découverte de nouveaux Musées par internet. S'il est possible d'aller les visiter, une sortie exploratoire sur le terrain est organisée. Les données recueillies par les élèves serviront à alimenter leur propre création artistique. Ils deviennent à la fois explorateurs, chercheurs, découvreurs, inventeurs et surtout créateurs de sens et d'objet esthétique. L'exercice est lui-même médiateur culturel, car il permet de découvrir de nouveaux musées comme le démontrent les exemples qui suivent.

\section{Résultats d'essais exploratoires du modèle : quelques exemples.}

En mettant en pratique le modèle d'enseignement arTSchives, nous avons découvert qu'une peinture devient un tremplin vers l'histoire lorsqu'une œuvre de Mario Doucette (artiste acadien) issue de l'exposition collective «Oh, Canada (en montre à la Owens Art Gallery, Sackville du 26 juin au 21 septembre 2014) est suivi d'une visite du Musée acadien de l'Université de Moncton où on découvre d'autres archives en lien avec l'histoire de la dispersion du peuple acadien. 
Une autre œuvre de l'exposition « Oh, Canada », une installation de Diane Landry (artiste québécoise), devient comme tremplin vers la science lorsque ses objets hétéroclites en mouvement et découvrir le nouveau Discovery Center d'Halifax en Nouvelle-Écosse.

L'œuvre «Le soleil » de Dale Chihuly (artiste américain), installée devant le Musée des Beaux-Arts de Montréal et visité sur place, est précédée du visionnement du montage de l'œuvre disponible en ligne sur le site internet du MBAM puis devient un tremplin vers des souffleurs de verre lorsqu'on visite le site internet du Musée The Corning Museum of Glass de l'état de New York.

À Moncton, une photographie d'archives montrant l'énorme cube de glace qui faisait partie du processus de création de l'Installation Le miroir aux alouettes de Jean-Yves Vigneault (artiste des Iles de la Madeleine), alors qu'il réalisait une installation in situ dans le cadre du Symposium d'art nature de l'Acadie 2004, devient un tremplin vers Le jardin des glaciers de Baie-Comeau au Québec.

De nouveaux Musées furent ainsi découverts grâce à des œuvres d'art archivistiques en mettant en application le modèle d'enseignement arTSchives, en allant visiter des Musées d'art contemporain et en utilisant les nouvelles technologies. De plus, des œuvres d'art contemporain prennent tout à coup un nouveau sens aux yeux de l'apprenant ou de l'apprenante suite à cette expérience d'enseignement des arts visuels.

\section{Conclusion}

Le modèle d'enseignement présenté dans cet article avait pour but de contribuer à l'appréciation de l'art archivistique tout en encourageant la créativité grâce à l'intégration d'archives dans les étapes du processus de création. Le but était d'améliorer les interventions éducatives en enseignement des arts visuels en lien avec l'appréciation et la réception de l'art 
contemporain. Par le fait même, nous espérions contribuer, par notre modèle d'enseignement, aux autres pistes de solutions proposées dans la littérature pour accompagner les jeunes vers l'appréciation de l'art contemporain. Cet objectif atteint, nous réalisons qu' ArTSchives s'ouvre sur une découverte et sur les pistes de découvertes que voici :

Par exemple, nous avons réalisé que les musées d'histoire, de sciences naturelles, de sciences humaines et autres types de musées, grâce aux artéfacts et objets divers, peuvent jouer un rôle de tremplin vers la compréhension de l'art du 21e siècle, car les artistes d'aujourd'hui s'inspirent de ces divers objets pour créer. Les technologies entrent aussi en ligne de compte dans ce processus car plusieurs musées ont maintenant leurs sites internet. Ce médium est utile à la fois pour identifier des archives, découvrir de nouvelles œuvres et autres lieux de diffusion de l'art contemporain. Il facilite la découverte d'art archivistique et de musées virtuels. Cela offre par le fait même une occasion aux élèves d'être des archivistes de leurs propres créations artistiques (ex. à l'aide de la caméra numérique et du portfolio électronique).

Suite à l'analyse des données, l'étude révèle une autre piste de recherche. Celle-ci est en lien avec la notion de l'immatériel en art contemporain (ex. dans le cadre d'art en direct : les échanges entres l'artiste et le public dans le processus de création). Nous remarquons que cette notion de l'immatériel présente des similarités avec l'histoire orale. Or il est connu que le peuple acadien a une longue tradition de l'histoire orale (voir Centre d'études acadiennes). Nous y décelons une piste à suivre qui consisterait à faire des liens entre l'éducation artistique, les archives acadiennes et l'art contemporain.

Une autre piste qui s'est révélée intéressante lors de cette étude est issue du processus méthodologique, celle en lien avec l'analyse de nos propres installations éphémères comme données visuelles pour en déceler des exemples d'archives, et qui consisterait à vérifier si l'usage 
de la stratégie archivistique amènerait les élèves, ainsi que leur enseignante ou enseignant, à découvrir, par leurs propres observations et vécu culturel, de nouveaux exemples d'archives utilisées, ou à explorer, en arts visuels. Il serait aussi intéressant de voir si les jeunes, avec leur connaissance des nouvelles technologies et de la culture populaire, ont plus de facilité à décoder les œuvres d'art archivistique que la génération précédente.

En dernier lieu, il faudrait faire une étude plus approfondie de l'application du modèle arTSchives. Il nous semble qu'il serait intéressant d'étudier de ce qui se passe lors des étapes du processus de création artistique avec une perspective archivistique. De même, l'application du modèle dans le contexte d'appréciation des œuvres d'art reste une piste à explorer plus profondément.

Cependant, nous constatons que les divers objets exposés dans les musées inspirent les artistes contemporains. Les œuvres d'art contemporain de notre époque en témoignent. Au Nouveau-Brunswick, par exemple, des artistes francophones, comme Marjolaine Bourgeois, y travaillent et plusieurs projets de collaboration ont lieu sur le terrain de l'Université de Moncton entre son musée historique et sa galerie d'art contemporain. Cet échange entre passé et présent et vice-versa est enrichissant. D'un musée à l'autre, une fréquentation de divers types de musées dans un esprit de découverte et de rêverie, voilà un terrain idéal pour s'initier à l'art contemporain de genre archivistique.

Pour terminer, nous croyons qu'en éducation artistique, la notion d'archives doit être intégrée dans l'enseignement des arts visuels au primaire et au secondaire afin de mettre le cours d'arts visuels à jour avec le $21^{\mathrm{e}}$ siècle. ArTSchives, en ce sens, est une piste de solution à considérer. Ce modèle d'enseignement, par la valorisation de la poésie dans le processus de lecture de l'œuvre et de création artistique, valorise la rêverie, la créativité et les idées des 
apprenantes et des apprenants. L'art devient un tremplin vers divers types de musées avec leurs milliers d'archives et vice-versa. On espère ainsi contribuer à l'enseignement des arts visuels et arts médiatiques en facilitant la compréhension d'une des formes d'arts visuels de notre époque : l'art archivistique.

\section{Références}

Duncum, P. (2001). Visual culture: Developments, definitions and directions for art education. Studies in Art Education 42(2), 101-112.

Feinstein H. (1989). The art response guide: How to read art for meaning, a primer for art criticism. Art Education, 42, 43-53.

Foster, H. (2004). An archival impulse. October, 110, 3-22.

Grande, John. (2000). Made in Québec. Art and the Quebecers. CIRCA. Irish and International Contemporary Visual Culture, 94 (winter), 17-21.

Klein, A., Lessard, D., \& Lacombe A.-M. (2014). Archives et mise en Archives dans le champ culturel. Dans Y. Lemay, \& A. Klein (Eds.). Archives et création: Nouvelles perspectives sur l'archivistique. Cahier 1. Montréal, QC: Université de Montréal, École de bibliothéconomie et des sciences de l'information (eBSi).

Klein, A., Lacombe A.-M., \& Lemay, Y. (Dir.) (2014). Archives et création, regards croisés sur les archives: Tournant archivistique, courant artistique. Colloque tenu lors du 82e Congrès de l'ACFAS, Montréal, Université Concordia, 16 mai. $<$ http://www.acfas.ca/evenements/congres/ programme/82/300/319/c > (consultée le 11 septembre 2015).

Kolb, D.A. (1984). Experiential Learning. Englewood Cliffs, NJ: Prentice-Hall 
Lachapelle, R. (2013). Controversies about public contemporary art: an opportunity for studying viewer responses. Canadian Review of Art Education, 40, 94-115.

Lemay, Y., \& Klein, A. (Dir.). (2014). Archives et création: Nouvelles perspectives sur l'archivistique. Cahier 1. Montréal, QC: Université de Montréal, EBSI. Repéré à http://hdl.handle.net/1866/11324

Lopez, X., Margapoti, I., Maragliano, R., \& Bove, G. (2010). The presence of Web 2.0 tools on museum websites: a comparative study between England, France, Spain, Italy and the USA. Museum Management and Curatorship, 25(2), 235-249.

Robichaud, L. (1990). Voir l'art. Des artistes acadiens en milieu scolaire francophone au Nouveau-Brunswick. Moncton, NB: Éditions Michel Henry.

Robichaud, L. (2000). "Liens". Installation in situ réalisée dans le cadre de Mer Océane: Symposium en arts visuels des iles de la Madeleine. Québec.

Robichaud, L. (2004). Création en arts médiatiques et transferts en éducation artistique (New media, children and visual art education.) In Proceedings international Conference on New Media Research Networks. UPEI: Charlottown, IPE, 27-32.

Robichaud, L. (2011a). Visual arts and mathematics: Intertwining for a new conversation in education. In B. Sriraman \& V. Freiman (Eds), Interdisciplinarity for the 21st Century: Proceedings of the 3rd International Symposium on Mathematics and its Connections to Arts and Sciences, Moncton 2009. Monograph 11 in The Montana Mathematics Enthusiast Monographs in Mathematics Education, Information Age Publishing, Charlotte, NC, 239-255.

Robichaud, L. (2011b). "Le Musée acadien: Une contribution unique au niveau de la culture et du patrimoine francophone dans la formation des maitres". Communication_dans le cadre 
de la journée d'étude : Les Canadiens et leurs passés [Alliance de recherche universitéscommunautés (A.R.U.C.)] et le Musée acadien de l'Université de Moncton (MAUM) le 20 janvier 2011 à l'Université de Moncton, Campus de Moncton.

Robichaud, L., \& Cormier, J.-M. (2013). "L’éducation au patrimoine culturel acadien" Symposium francophone 2013 (partie I: Robichaud: contexte théorique; partie II. Cormier: exemples d'activités du musée). Moncton, NB: Université de Moncton.

Walker, D. (1990). Fundamentals of Curriculum. New York : Harcourt Brace Jovanovich.

White, B. (2014). Student generated art criticism. Canadian Review of Art Education, 40, 56-76. 